\title{
Successful conservative management of a rare case of ruptured cornual ectopic pregnancy
}

\begin{abstract}
Cornual pregnancy is a rare type of ectopic pregnancy with incidence of about $2-4 \%$ of all ectopic pregnancies. The mortality rate is about $2-2.5 \%$. The diagnosis and management is challenging and frequently constitute a medical emergency. We report a case of a 33year old second gravida, para 1, living 1 with previous LSCS with 13weeks 2days period of gestation presenting to the casualty with complaints of severe pain abdomen and signs of hypovolemic shock. Ultrasound revealed gross hemoperitoneum with ruptured right adnexal gestation. Emergency laparotomy was done and cornual end was found ruptured and was sutured successfully and hemostasis achieved. Intra operative two units of packed cells was transfused. Patient was discharged stable on post-op day 7.
\end{abstract}

Keywords: ectopic pregnancy, hypovolemic shock, hemoperitoneum, laparotomy, blood transfusion
Volume 8 Issue I - 2020

\author{
Sumayya Sana, Pruthvi S, Sreelatha S, \\ Yashaswini, Shruthi K, Shridhar S \\ Department of Obstetrics and Gynecology, ESIC Medical \\ College \& PGIMSR, India
}

Correspondence: Sreelatha S, Department of Obstetrics and Gynecology, ESIC Medical College \& PGIMSR, India, Email dr.sreelath20II@gmail.com

Received: October 16, 2019 | Published: February 24, 2020

\section{Introduction}

About $6-16 \%$ of all pregnant women who present to an emergency obstetric department with first-trimester bleeding or pain or both have ectopic pregnancy. Due to the nature of pathology of ectopic pregnancy and the absence of a single diagnostic test, early diagnosis can be often difficult. An ectopic or extrauterine pregnancy is one in which the blastocyst implants anywhere other than the endometrial lining of the uterine cavity. Nearly $95 \%$ of ectopic pregnancies implant in the fallopian tube. The sites of ectopic pregnancy include ampullary part of fallopian tube (70\%), isthmic part (12\%), fimbrial part (11\%), ovarian (3\%), interstitial and cornual part (2-3\%), abdominal (1\%), caesarean scar and cervical $(<1 \%) .{ }^{1}$ The most important risk factor of an ectopic pregnancy is a prior ectopic pregnancy. Other risk factors include tubal surgery, pelvic infection, infertility and treatment, smoking, women exposed to diethylstilbestrol in utero, use of contraception especially intra-uterine device, sterilization failure, etc. Recognition of high-risk cases, early diagnosis (even before rupture) with the use of TVS, serum $\beta$-hCG and laparoscopy have significantly improved the management of ectopic pregnancy.

The parts of fallopian tube are fimbrial, ampullary, isthmic and interstitial part. The interstitial part is the narrowest and proximal portion of the fallopian tube that lies within the muscular wall of the uterus. It is $0.7 \mathrm{~mm}$ wide and approximately $1-2 \mathrm{~cm} \mathrm{long}$, with a slightly tortuous course extending obliquely upwards and outwards from the uterine cavity. Pregnancies implanted in this site are called as interstitial or cornual pregnancies. The term cornual pregnancy remains ambiguous as it is also used to indicate pregnancy located within the cavity in one of the two upper horns of a bicornuate uterus. Risk factors are similar to other ectopic pregnancies, although prior ipsilateral salpingectomy is a specific risk factor for interstitial pregnancy. ${ }^{2}$ A cornual pregnancy is rare and one of the most dangerous type of ectopic pregnancy since rupture of this pregnancy occurs late due to thick and vascular musculature wall with greater distensibility, the fetus grows dissecting the muscle fibres for a longer period (12-14weeks). Significant hemorrhage occurs since this interstitial part is supplied by the Sampson artery, which represents the anastomosis of uterine and ovarian artery, and due to increased vascularity of the sac due to greater period of gestation. It is associated with the mortality rate as high as $2.5 \%$, which is 6 to 7 times higher than other ectopic pregnancies in general.

Because of its location, early diagnosis of cornual pregnancy is difficult. The eccentric position of the gestational sac and thinning of the myometrial mantle means that differentiation between eccentric intrauterine and cornual pregnancy is often difficult. ${ }^{3}$ Asymmetrical enlargement of the uterus especially during contraction is a conspicuous finding. It can be confused with lateral flexion of a gravid uterus, pregnancy associated with fibroid, pregnancy in a bicornuate uterus or angular pregnancy.

An early diagnosis is important and is facilitated by the use of high resolution ultrasonography and the quantitative HCG assay. The USG criteria for making a diagnosis includes:

i. An empty uterus;

ii. A gestational sac seen separately and $<1 \mathrm{~cm}$ from the most lateral edge of the uterine cavity and;

iii. A thin myometrial layer surrounding the sac, $<5 \mathrm{~mm} .{ }^{4}$

The gestational sac is usually in the lateral portion of the uterus early in gestation but in advanced comual pregnancy it can be located above the uterine fundus and can be confused with an eccentric intrauterine pregnancy. This is referred to as the 'interstitial line' sign. A thin echogenic line extends directly up to the centre of the cornual gestational sac: this represents either the endometrial cavity or the interstitial portion of the fallopian tube, depending on the size of the cornual pregnancy. ${ }^{4}$ The last criteria having the greatest diagnostic sensitivity $(80 \%)$ and specificity $(98 \%){ }^{5}$

Traditionally, the management of a cornual pregnancy has been 
hysterectomy or cornual resection at laparotomy. This was associated with morbidity and unfavorable effects on future fertility, more conservative approaches have been introduced recently into clinical practice. Medical treatment with methotrexate has been successfully used in early diagnosed cornual pregnancy.

\section{Case report}

A 33year old, second gravida, para one, living one, with previous LSCS with 13 weeks 2 days period of gestation presented to our casualty with acute onset of severe lower abdominal pain since 4hours, associated with breathlessness. On examination she was moderately built and moderately nourished with severe pallor and signs of shock with pulse rate of $110 \mathrm{bpm}$, blood pressure of $90 / 60 \mathrm{mmHg}$. Systemic examination revealed tender and distended abdomen. Vaginal examination revealed cervical motion tenderness, uterine size was not made out because of distended abdomen, and forniceal tenderness was seen. Laboratory investigations were done, all were found normal, except for haemoglobin which showed $6 \mathrm{~g} / \mathrm{dL}$. Emergency ultrasound was done which revealed a gestational sac of 12 weeks occupying right adnexa and absent FHR, fundus of uterus was ill-defined, with empty uterine cavity with significant hemperitoneum. Impression was? Tubal/? Cornual ruptured pregnancy.

Emergency laparotomy was done under general anesthesia. There was about $2700 \mathrm{ml}$ of blood with clots and fetus with placenta was found in the peritoneal cavity. Right cornual end was found ruptured and bilateral tubes and ovaries were seen separately, normal from it. The cornual end was repaired successfully with vicryl 1 in intermittent sutures and hemostasis achieved. Intra-op she was transfused with 2 units of packed cells. She was shifted to ICU post-operatively for observation. 2days later she was shifted to ward and was discharged on day 7 in a stable condition. Counselling regarding future fertility was done (Figures 1-4).

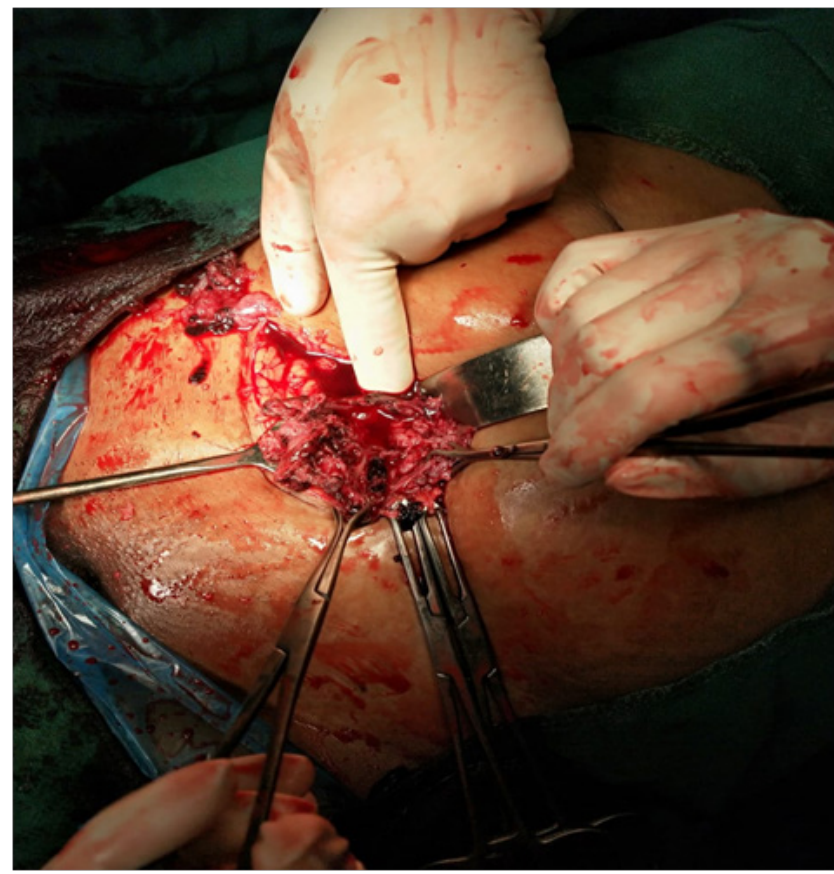

Figure I Ruptured right cornual end.

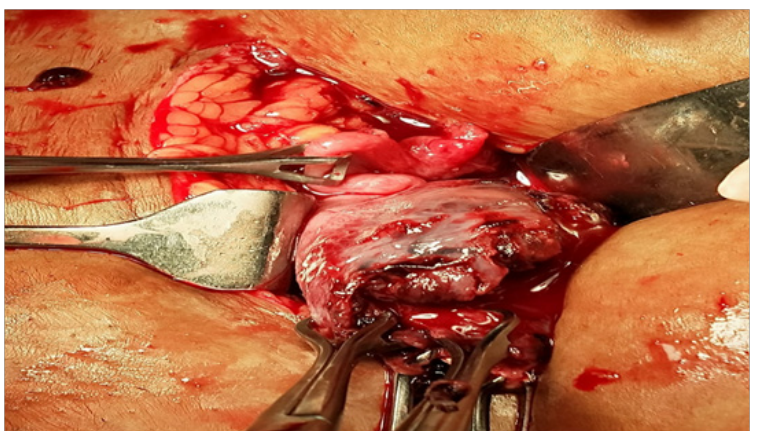

Figure 2 Bilateral tubes and ovaries seen seperately from ruptured cornual end.

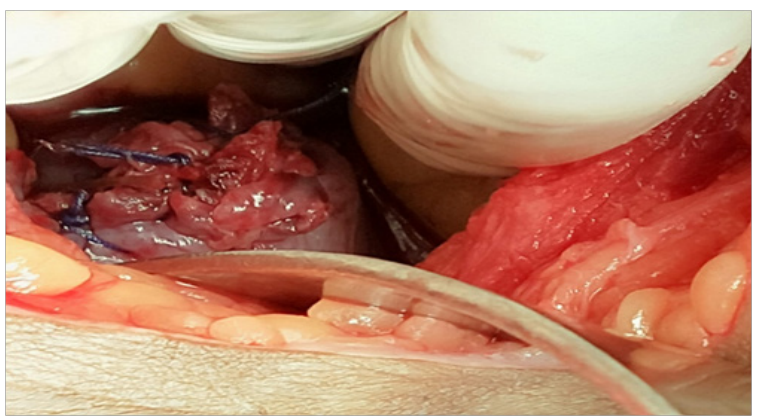

Figure 3 Ruptured cornual end sutured successfully.

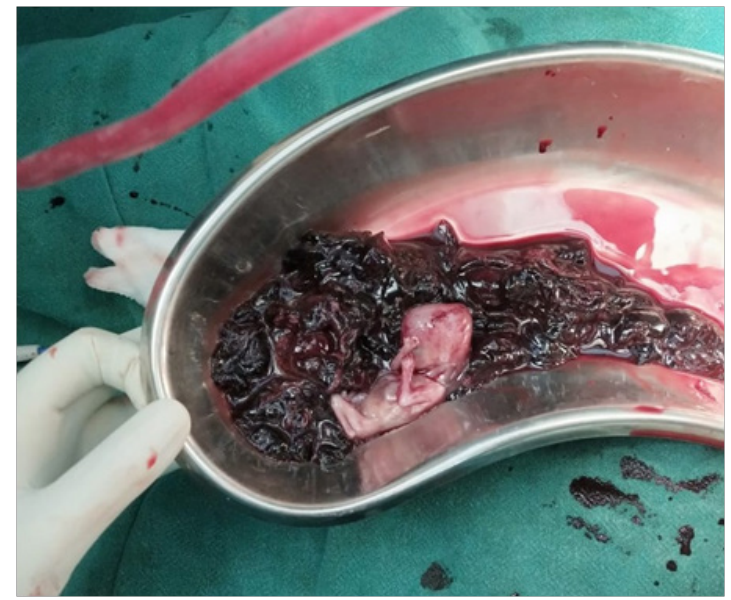

Figure 4 Fetus with blood clots removed from peritoneal cavity.

\section{Discussion}

Cornual gestation is one of the most hazardous types of ectopic gestation. The diagnosis and treatment is challenging and frequently constitute a medical emergency. It has a mortality rate which is 6-7times higher than that of the ectopics in general. ${ }^{6}$ Traditionally, the treatment of cornual pregnancy has been hysterectomy or cornual resection at laparotomy. As all surgical management has been associated with morbidity and unfavorable effects on future fertility, more conservative approaches have been introduced into clinical practice. Medical treatment (as with other types of tubal pregnancy) has been introduced with generally satisfactory results. Faraj R et 
al., in his observational study concluded that early clinical diagnosis with ultrasound or laparoscopy may help to contribute towards effective conservative management. This includes laparoscopic conservative treatment and medical treatment with systemic methotrexate. Sonographically guided, minimally invasive treatment can be a safe and effective alternative to surgical and systemic medical therapy. ${ }^{4}$

According to Faraj $\mathrm{R}$, et al, management options for cornual ectopic pregnancy includes: ${ }^{4}$

\section{a. Surgical}

\section{Laparotomy:}

i. Hysterectomy;

ii. Cornual resection;Uterine artery ligation \& repair of ruptured uterine cornu.

\section{Laparoscopic procedures:}

i. Cornual resection;

ii. Salpingostomy;

iii. Cornual resection and salpingectomy;

iv. Endoloop and encircling suture. ${ }^{7}$

\section{Hysteroscopic procedures:}

i. Hysteroscopic endometrial resection under laparoscopic control;

ii. Hysteroscopic cornual evacuation aided by polyp forceps under ultrasound (USS) or laparoscopic guidance.

\section{b. Medical}

i. Systemic methotrexate;

ii. USS guided methotrexate;

iii. Laparoscopic guided methotrexate/potassium chloride;

iv. Systemic methotrexate followed by selective uterine artery embolisation $^{8}$

Shiragur SS et al.9 reported case of ruptured cornual ectopic gestation at 8 weeks which was successfully managed by cornual end repair. ${ }^{9}$ Peterson et al concluded that physicians should consider ectopic pregnancy when attempts at induced mid-trimester abortion do not succeed. ${ }^{10}$

Khawaja et al.11 reported a unique method of management for ruptured cornual gestation, using uterine artery ligation to conserve the uterus. The authors suggested that ipsilateral uterine artery ligation should be performed before attempting to repair a ruptured uterine cornu. This will help to achieve hemostasis and allow time to repair the ruptured cornual end. ${ }^{11}$

Dziadosz $\mathrm{M}$ et al,12 reported a case of interstitial pregnancy of 10weeks managed conservatively with local injection of methotrexate. As per protocol, under ultrasound guidance with the assistance of an automated puncture device, a 21-gauge needle was introduced into the gestational sac. Half of the prepared $100 \mathrm{mg}$ of methotrexate in $2 \mathrm{ml}$ of solvent was injected. The puncture site was monitored for postprocedure bleeding. The needle should pass through the myometrium to allow the point of least resistance to empty into the cavity and thereby avoiding leakage or rupture into the abdomen. Morison's pouch was inspected further and hemostasis was ensured. The patient experienced 2 weeks of progressively decreasing vaginal bleeding and declining BhCG without complications. ${ }^{12}$

One of the concerns of future pregnancy is rupture of the interstitial portion of the tube (uterine rupture). The postulated mechanism is through a defective area of uterine wall. ${ }^{13}$ The second concern after conservative management of cornual pregnancy is recurrence of ectopic pregnancy, particularly cornual pregnancy on the same side. Tubal pathology is often the primary factor blamed for recurrence. It is therefore important to counsel appropriately regarding future pregnancy risks and plan optimum mode of delivery.

\section{Conclusion}

Cornual pregnancy has a significant diagnostic and therapeutic challenge. It posses a greater maternal morbidity and mortality compared to other ectopic pregnancy in general. Early and suspicious clinical diagnosis and careful ultrasonography may help to contribute towards effective conservative management and reduce mortality. Appropriate individual counselling is needed regarding risks of future pregnancy and mode of delivery.

\section{Acknowledgments}

None.

\section{Conflicts of interest}

The authors declare that there is no conflict of interest.

\section{Funding}

None.

\section{References}

1. Bouyer J, Coste J, Fernandez H, et al. Sites of ectopic pregnancy: a 10year population-based study of 1800 cases. Hum Reprod. 2002; 17(12):3224-3330

2. Lau S, Tulandi. Conservative medical and surgical management of interstitial ectopic pregnancy. Fertil Steril. 1999;72(2):207-215.

3. Auslender R, Arodi J, Pascal B, et al. Interstitial pregnancy: earlydiagnosis by ultrasonography. Am J Obstet Gynecol. 1983;146(6):717-718.

4. Radwan Faraj, Martin Steel. Management of cornual (interstitial) pregnancy. Royal College of Obstetricians and Gynaecologist. 2007;9(4):249-255.

5. Fritz, Marc A, Leon Speroff. Clinical Gynecologic Endocrinology and Infertility. $8^{\text {th }}$ edn. Philadelphia: Wolters Kluwer Health/Lippincott Williams \& Wilkins; 2011.

6. Surekha SM. A ruputured left cornual pregnancy: a case report. Journal of clinical and diagnostic research: JCDR. 2013;7(7):1455-1456.

7. Reich H, Johns DA, DeCaprio J, et al. Laparoscopic treatment of one hundred and nine consecutive ectopic pregnancies. J Reprod Med. 1988;33:885-890.

8. Kok-Min S, Bin-Chwen Hsieh YL-A, Tsai L-W, et al. Expectant management of a cornual pregnancy followed up by serial transvaginal color power Doppler angiography and serum beta human chorionic gonadotropin levels. Acta Obstet Gynecol Scand. 2004;83(12):12211224 . 
9. Shiragur SS, Gobbur RV, Tehalia JM, et al. Ruptured cornual ectopic pregnancy at 8 weeks gestation- successful conservative approach: a case report. Int J Reprod Contracept Obstet Gynecol. 2013;2(4):671673.

10. Petersen KR, Larsen GK, Nørring K, et al. Misdiagnosis of interstitial pregnancy followed by uterine cornual rupture during induced midtrimester abortion. Acta Obstet Gynecol Scand. 1992;71(4):316-318.

11. Khawaja N, Walsh T, Gill B. Uterine artery ligation for the management of ruptured cornual ectopic pregnancy. Eur J Obstet Gynecol Reprod Bio. 2005;118(2):269.
12. Dziadosz M, Monteagudo A, Timor-Tritsch I. Interstitial pregnancy. In: Tulandi T, Editor. Ectopic Pregnancy. New York: Springer; 2015. p. $77-84$.

13. Dubuisso JB, Fauconnier A, Babaki-Fard K, et al. Laparoscopic myomectomy: a current view. Hum Reprod Update. 2000;6(6):588-594. 